\title{
Appel pour la saisie des études cliniques dans un registre accessible au public
}

Les signataires de cette invitation s'engagent pour qu'en Suisse - comme dans d'autres pays - chaque essai clinique soit saisi dans un registre accessible au public. Tant pour des raisons éthiques que scientifiques, un tel registre paraît indispensable; il garantit une information complète sur l'état des connaissances scientifiques et offre aux médecins et aux patients un meilleur accès aux informations relatives aux possibilités de traitements. Le caractère global de la recherche clinique justifie qu'un tel projet doit être réalisé en étroite collaboration internationale.

Cet appel est soutenu par

Prof. Peter M. Suter,

Président de l'ASSM, Genève

Prof. Werner Stauffacher,

Viceprésident de l'ASSM, Bâle

Prof. Peter Meier-Abt,

Viceprésident de l'ASSM, Zurich

Dr. Jacques de Haller

Président FMH, Berne

Prof. Dieter Imboden,

Président du Conseil de la recherche du Fonds national suisse, Zurich

Prof. André Perruchoud,

Président du "Collège des Doyens»

des Facultés de médecine suisses, Bâle

ainsi que les participants $d u$ «Rencontre des représentants des institutions du domaine des essais cliniques", 26 janvier 2005, Berne
Toutes les études cliniques réalisées sur des êtres humains sont soumises à des lois, des standards et des principes éthiques nationaux et internationaux dont le but est de garantir la protection des sujets de recherche et la validité des données. A cet égard, il est important que les connaissances acquises grâce à l'engagement personnel des patients soient à la disposition illimitée du système de santé et servent à la planification et au déroulement des études qui suivent.

Par la publication retardée ou dissimulée des études interrompues ou des études dont les résultats sont indésirables ou présumés inintéressants (des études dites négatives), les informations et connaissances escomptées peuvent être limitées. Une évaluation erronée - la plupart du temps trop optimiste - d'un médicament ou d'un procédé non médicamenteux peut en résulter. En outre, le fait d'ignorer que des études ont été planifiées ou réalisées peut avoir des effets négatifs considérables, lorsque par exemple, les risques de certains procédés médicaux sont méconnus.

Grâce aux registres d'études cliniques, de telles répercussions négatives pourraient être évitées et l'acquisition de connaissances encouragée

- en informant les médecins et les patients ainsi que les institutions du système de santé des différentes études en cours, ce qui permettrait d'accélérer leur déroulement en facilitant l'admission des patients;

- en offrant au public intéressé une information transparente sur les études cliniques;

- en publiant les problèmes et les expériences liés aux études et en en tenant compte lors de la planification de nouvelles études;
- en évitant les études redondantes et en permettant ainsi le déroulement d'autres études importantes;

- en promouvant la coopération entre les personnes effectuant des études et en accédant à une meilleure force d'expression grâce à des études plus importantes élaborées en commun.

Pour toutes ces raisons, il devient de plus en plus urgent de saisir toutes les études cliniques dans un registre accessible au public. Plusieurs organisations nationales et internationales ainsi que des éditeurs de revues scientifiques ont déjà adhéré à cette demande et enregistrent leurs études ou ne publient que les études enregistrées. A ce jour, il n'existe en Suisse que peu d'initiatives isolées allant dans ce sens, mais aucun pas vers une saisie systématique de toutes les études n'a été entrepris. Récemment, lors de la révision de l'ordonnance sur les essais cliniques avec des médicaments, en dépit des demandes émanant de divers milieux (de l'Académie Suisse des Sciences Médicales et de la Société Suisse de l'Industrie Chimique entre autres) le Conseil Fédéral a renoncé à y introduire de telles prescriptions.

Les signataires de cette invitation demandent aux instances politiques de rendre obligatoire la saisie des études cliniques dans un registre accessible au public, dans les plus brefs délais. A cet égard, il s'agit de vérifier si la création d'un registre suisse s'impose (en étroite coordination et coopération avec les registres internationaux) ou si l'adhésion à un registre international existant est préférable.

Dans l'intervalle, les signataires demandent à tous les responsables d'études ainsi qu'à tous les promoteurs publics et privés de s'assurer, dans le cadre de leurs fonctions et de leurs possibilités, que les études cliniques réalisées en Suisse soient enregistrées dans les registres existants. En outre, ils encouragent tous les autres participants, comme par ex. les commissions d'éthique, les autorités d'admission et les responsables des instituts de recherche, chacun dans son domaine respectif, à faire enregistrer systématiquement toutes les études cliniques. 